\title{
Uncommon uses for a common fish
}

\author{
by Gregory D. Larsen
}

\section{SCIENTIFIC NAME \\ Oryzias latipes}

\section{TAXONOMY}

PHYLUM: Chordata

CLASS: Actinopterygii

ORDER: Beloniformes

FAMILY: Adrianichthyidae

\section{General description}

Medaka, also known as Japanese rice fish, is an egg-laying, bony fish that is native to freshwater and brackish habitats in parts of China, Korea and Japan. Medaka are small fish, reaching a standard length of about $2.5-3.0 \mathrm{~cm}$ at sexual maturity. They are hardy, resistant to many common diseases ${ }^{1}$ and able to survive temperatures as extreme as $0{ }^{\circ} \mathrm{C}$ and $40^{\circ} \mathrm{C}$ (ref. 2). These characteristics make them wellsuited to captivity, and medaka have been kept and bred as pets in Japan for hundreds of years.

\section{Husbandry}

Medaka is a particularly convenient species for laboratory research. Besides their resilient physiology, medaka breed and grow quickly, with a generation time of only $6-8$ weeks ${ }^{1}$. Females produce $30-50$ eggs per day and up to 3,000 eggs in a mating season, which can be spawned unfertilized if medaka females are mated with sterile hybrid males. Eggs can be fertilized in vitro within 8 hours of spawning, and embryos can be kept at low temperatures to slow development for up to 3 months. Embryos can be raised at a maximum density of 200 eggs per 9-cm Petri dish, and embryos develop into feeding young adults within 7 days. In strains with sex-linked pigmentation patterns, individual embryos can even be sexed within their transparent eggs by the second day after fertilization.

\section{Research résumé}

Medaka have a long and distinguished history as a model species for research. In the early 1900s, Japanese researchers used medaka to establish Mendelian inheritance in fish, and in 1921, medaka was used to demonstrate sex-linked inheritance in animals ${ }^{3}$. Medaka were subsequently used for many studies of sex determination and differentiation, and during the late 1900s they were a popular animal model in genetics. Medaka remain very useful for genetic work today, with one of the smallest genomes among vertebrate genetic models, at an estimated 650-1,000 Mb. The zebrafish genome, by comparison, is roughly twice as large, and that of humans is about three times as large ${ }^{1}$.

There are now many transgenic lines of medaka for use in research, and scientists have created powerful models by combining visually transparent strains with transgenic lines that express green fluorescent protein ${ }^{4}$. Researchers have used these models to track and monitor specific cells and organs throughout embryonic development, growth and adulthood under various experimental conditions. This is especially valuable in toxicology, where researchers can now visualize precisely how chemicals alter growth and physiology. Today, both transgenic and wild-type medaka are used as common models in toxicology and environmental monitoring of aquatic habitats ${ }^{5,6}$.

In 1994 medaka also became the first vertebrate species to mate in space. In an experiment of the Second International Microgravity Laboratory aboard the space shuttle Columbia, medaka success-

fully mated, laid eggs and hatched under microgravity ${ }^{7}$. Subsequent studies examined the development and swimming behavior of medaka fry during spaceflight $^{8}$, and in 2012, the Japanese Space Agency sent an aquarium, called the Aquatic Habitat, to the international space station to support experiments with zebrafish and medaka. A new publication from one such experiment reports high osteoclast activity and bone resorption in transgenic medaka reared in the space station's microgravity environment ${ }^{9}$. These findings parallel observed decreases of bone mineral density in astronauts, and it is hoped that further experiments with medaka can clarify how microgravity alters the physiology and overall health of vertebrates during spaceflight.

1. Wittbrodt, J., Shima, A. \& Schart, M. Medaka-a model organism from the far east. Nat. Rev. Genet. 3, 53-64 (2002).

2. Shima, A. \& Hiroshi, M. Medaka as a research organism: past, present and future. Mech. Dev. 121, 599-604 (2004).

3. Naruse, K., Tanaka, M. \& Takeda, H. (eds.) Medaka: A Model for Organogenesis, Human Disease, and Evolution (Springer, Tokyo, 2011).

4. Tanaka, M., Kinoshita, M., Kobayashi, D. \& Nagahama, Y. Establishment of medaka (Oryzias latipes) transgenic lines with the expression of green fluorescent protein fluorescence exclusively in germ cells: A useful model to monitor germ cells in a live vertebrate. Proc. Nat. Acad. Sci. 98, 2544-2549 (2001).

5. Kinoshita, M. Transgenic medaka, small fresh water teleost (Oryzias latipes), is now available for environmental science. Int. Stud. Environ. Chem. 6, 49-54 (2012).

6. Padilla, S. et al. Use of medaka in toxicity testing. Curr. Protoc. Toxicol. 39, 1.10.1-1.10.36 (2009).

7. Ijiri, K. Explanations for a video version of the first vertebrate mating in space-a fish story. Biol. Sci. Space 11, 153-167 (1997).

8. Niihori, M., Mogami, Y., Kiyoshi N. \& Baba, S.A. Development and swimming behavior of medaka fry in a spaceflight aboard the Space Shuttle Columbia (STS-107). Zoolog. Sci. 21, 923-931 (2004).

9. Chatani, M. et al. Microgravity promotes osteoclast activity in medaka fish reared at the international space station. Sci. Rep. 5, 14172 (2015). 